\title{
Analysis of the dynamic response in the railway vehicles to the track vertical irregularities. Part II: The numerical analysis
}

\author{
M. Dumitriu \\ Department of Railway Vehicles, Faculty of Transport, University Politehnica of Bucharest, 313 Splaiul Independenţei, 060042, \\ Bucharest, Romania
}

Received 18 June 2015; Accepted 18 November 2015

\begin{abstract}
The paper examines the dynamic response of a two-bogie vehicle to the symmetrical and antisymmetrical excitations, due to bounce and pitch of the axles' planes, derived from the track vertical irregularities. Part I introduced the theoretical model and the response functions of the vehicle, as well as the theoretical elements required for the analysis of the dynamic response of the vehicle to the track stochastic irregularities. Part II comprises the results of the numerical analysis of the vehicle dynamic response in three reference points of the carbody, based on which a series of properties of the vertical vibrations behaviour of the railway vehicle is pointed out at. The excitation modes that trigger the carbody response in its reference points are identified. Hence, the influence of the geometrical filtering effect of the excitation modes upon the ride quality and ride comfort is established.
\end{abstract}

Keywords: railway vehicle, response functions, geometric filtering effect, ride quality, ride comfort

\section{Introduction}

The dynamic response of the railway vehicle to the excitations due to the track irregularities can be examined underlining on the results derived experimentally or from numerical simulations. Even though the results of the line testing reflect the real exploitation conditions, carrying out the tests requires consistent financial and time investments. Moreover, such results can be impacted by a series of out-ofcontrol variables. The advantage of the numerical simulations is that a simple change in the numerical simulation parameters allows the examination of the dynamic response for various types of vehicles, under various exploitation conditions, even in the vicinity of certain extreme situations that cannot be distinguished in real testing circumstances [1 - 3]. The numerical simulations are used as basic tools in research and the designing stage for estimating the dynamic behaviour of the railway vehicle and the optimization of its dynamic performance, and later on for examining the issues emerging during exploitation. In the recent years, the specifications regarding the homologation of the railway vehicles from the perspective of the dynamic behaviour in terms of safety, track fatigue and ride quality [4, 5] have been added to a string of requirements so as to carry out the tests based on numerical simulations, thus regulating the so-called procedure of ,virtual homologation' [6, 7].

The analysis of the dynamic response of the vehicle to the track vertical irregularities is carried out in the paper via the numerical simulations developed on the vehicle model introduced in the Part I, where the inputs are given by the symmetrical and antisymmetrical excitation modes, coming

* E-mail address: madalina.dumitriu@yahoo.com ISSN: 1791-2377 @ 2015 Kavala Institute of Technology. All rights reserved. from the bounce and pitch motions of the axles' planes. As a matter of fact, the response functions in three reference points of the carbody are examined - at the centre and above the two bogies - and the partial response functions corresponding to the carbody movements to the symmetrical/antisymmetrical excitation modes, due to the bounce/pitch in the axles' planes. The dynamic response of the vehicle to the track stochastic irregularities is reviewed as based on the power spectral density of the carbody vertical acceleration. Similarly, the geometric filtering effect features of the four excitation modes are shown and the matching filtering speeds are examined. The root mean square of the vertical acceleration and of the comfort index to the vertical vibrations as speed function help emphasize the influence of the geometric filtering effect upon the ride quality and the ride comfort.

\section{The vehicle numerical model}

The parameters of the vehicle used in the numerical simulations are introduced in the table 1 . The table 2 includes the natural frequencies pertinent to the symmetrical and antisymmetrical motions of the vehicle, which have typical values for a passenger vehicle [8].

Table 1. The parameters of the vehicle's numerical model

\begin{tabular}{l|l}
\hline$m_{c}=34000 \mathrm{~kg}$ & $2 k_{z c}=1.2 \mathrm{MN} / \mathrm{m}$ \\
$m_{b}=3200 \mathrm{~kg}$ & $2 k_{x c}=340 \mathrm{kN} / \mathrm{m}$ \\
$m_{m 2}=35224 \mathrm{~kg}$ & $2 c_{z c}=34.44 \mathrm{kNs} / \mathrm{m}$ \\
$m_{m 3}=33950 \mathrm{~kg}$ & $2 c_{x c}=50 \mathrm{kNs} / \mathrm{m}$ \\
$J_{c}=1963840 \mathrm{~kg} \cdot \mathrm{m}^{2}$ & $c_{m 2}=53,469 \mathrm{kNm} / \mathrm{s}$ \\
$J_{b}=2048 \mathrm{~kg} \cdot \mathrm{m}^{2}$ & $c_{m 3}=142,052 \mathrm{kNm} / \mathrm{s}$ \\
$E I=3,2 \cdot 10^{9} \mathrm{Nm}^{2}$ & $4 k_{z b}=4.4 \mathrm{MN} / \mathrm{m}$ \\
$L=26.4 \mathrm{~m}$ & $4 c_{z b}=52.21 \mathrm{kNs} / \mathrm{m}$ \\
$2 a_{c}=19 \mathrm{~m} ; 2 a_{b}=2.56 \mathrm{~m}$ & $k_{m 2}=90,182 \mathrm{MN} / \mathrm{m}$ \\
$h_{c}=1.3 \mathrm{~m} ; h_{b}=0.2 \mathrm{~m}$ & $k_{m 3}=660,415 \mathrm{MN} / \mathrm{m}$ \\
\hline
\end{tabular}


Table 2. The natural frequencies of the symmetrical and antisymmetricl motions of the vehicle

\begin{tabular}{|c|c|c|c|}
\hline Symmetrical motions & 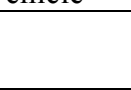 & $\begin{array}{l}\text { Antisym } \\
\text { motions }\end{array}$ & etrical \\
\hline Carbody bounce & $1.17 \mathrm{~Hz}$ & $\begin{array}{l}\text { Carbody } \\
\text { pitch }\end{array}$ & $\begin{array}{l}1.46 \\
\mathrm{~Hz}\end{array}$ \\
\hline Carbody bending & $8.20 \mathrm{~Hz}$ & $\begin{array}{l}\text { Carbody } \\
\text { bending }\end{array}$ & $\begin{array}{l}22.25 \\
\mathrm{~Hz}\end{array}$ \\
\hline Bogie bounce & $6.61 \mathrm{~Hz}$ & $\begin{array}{l}\text { Bogie } \\
\text { bounce }\end{array}$ & $\begin{array}{l}6.66 \\
\mathrm{~Hz}\end{array}$ \\
\hline Bogie pitch & $9.63 \mathrm{~Hz}$ & $\begin{array}{l}\text { Bogie } \\
\text { pitch }\end{array}$ & $\begin{array}{l}9.61 \\
\mathrm{~Hz}\end{array}$ \\
\hline
\end{tabular}

\section{The analysis of the vehicle response functions}

This section studies the vehicle response at the carbody level in its reference points - at the centre and above the two bogies, during the symmetrical and antisymmetrical excitation modes induced by the track vertical irregularities, as well as the geometric filtering effect characteristics of these excitation modes.

The figure 1 presents the partial response functions at the carbody centre, where the peaks corresponding to the natural frequencies of the carbody symmetrical bounce and pitch are pointed out at. The carbody response to the excitation coming from the symmetrical bounce of the axles' planes (diagram a) is dominated by the bounce motion $(1.17 \mathrm{~Hz})$. But when the vehicle is excited by the symmetrical pitch of the axles' planes (diagram (b)), the carbody response is then dominated by the symmetrical bending $(8.20 \mathrm{~Hz})$. The influence of the bogie bounce and pitch cannot be identified at the carbody level, due to the suspension damping. What should be noticed is the significant importance that the bounce motion has at the carbody centre, compared to the symmetrical bending.
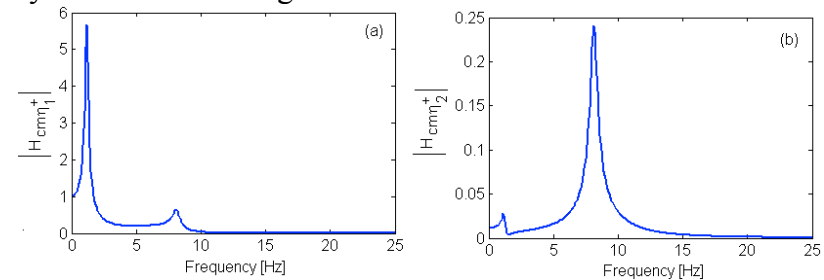

Fig. 1. The partial response functions at the carbody centre.

Figure 2 features the partial response functions in the carbody reference points located above the bogies. According to the diagrams (a) and (b), the vehicle carbody has a symmetrical bounce and bending motion during the symmetrical excitation modes from the bounce and the pitch in the axles' planes. The antisymmetrical bounce in the axles' planes excites the carbody pitch motion, as seen in diagram (c). Should the excitation is due to the antisymmetrical pitch in the axles' planes (diagram (d)), the response function has three peaks that correspond to the natural frequencies of the carbody pitch motion $(1.46 \mathrm{~Hz})$, its antisymmetrical bending $(22.25 \mathrm{~Hz})$ and the bogie pitch motion $(9.61 \mathrm{~Hz})$. Upon comparing the values in those four diagrams, the conclusion is that the dominant vibration modes are the carbody bounce and pitch in its reference points above the bogies.
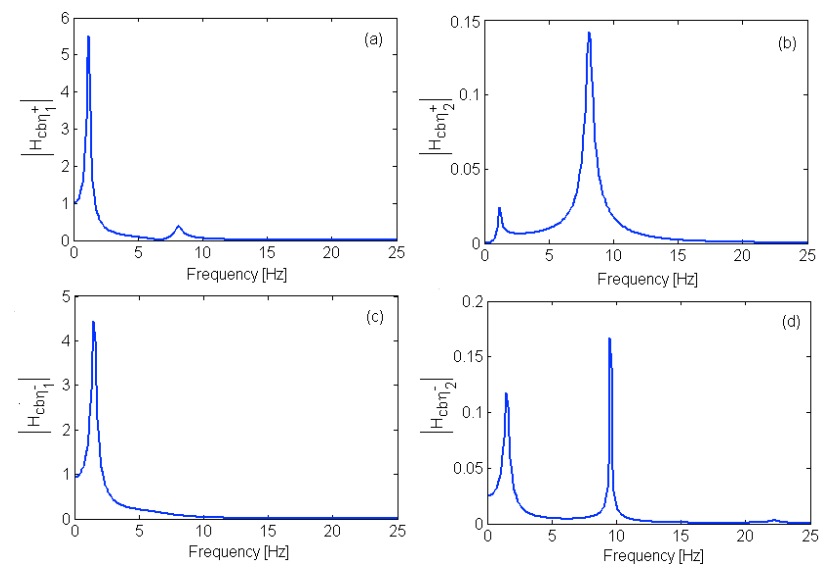

Fig. 2. The partial response functions of the vehicle above the bogies.

Figure 3 shows the geometric filtering effect characteristics of the symmetrical and antisymmetrical excitation modes at the resonance frequencies of the main carbody motions - bounce $(1.17 \mathrm{~Hz})$, symmetrical bending $(8.20 \mathrm{~Hz})$ and the pitch $(1.46 \mathrm{~Hz})$. They indicate the filtering speeds, namely the speeds for which the excitations from the bounce and pitch in the axles' planes are completely filtered ( $\bar{H}_{f 1,2}^{ \pm}=0$ ). On the one hand, it should be noticed that the geometric filtering effect is more efficient at frequency of $8.20 \mathrm{~Hz}$, where it presents more filtering speeds than in the other two similar cases. On the other hand, the geometric filtering effect can be noted to have a selective nature, depending on the velocity, more visible at low speeds. The higher the velocity, the larger the interval between two consecutive filtering speeds $[9,10]$.
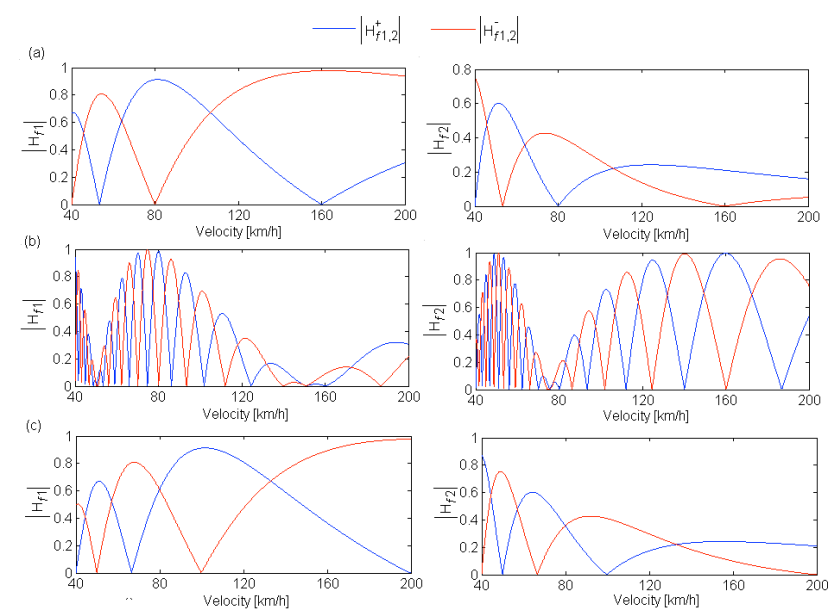

Fig. 3. The geometric filtering effect characteristics of the symmetrical and antisymmetrical excitation modes: (a) at frequency of $1.17 \mathrm{~Hz}$; (b) at frequency of $8.20 \mathrm{~Hz}$; (c) at frequency of $1.46 \mathrm{~Hz}$.

The filtering speeds can be calculated when starting from the relations (32) - (33) introduced in Part I of the paper herein, which define the geometric filtering effect characteristics of the excitation modes corresponding to the bounce and pitch in the axles' planes. They will write as below:

- for the symmetrical excitation modes

$\bar{H}_{f 1}^{+}=\cos \frac{\omega}{V} a_{b} \cos \frac{\omega}{V} a_{c} ; \quad \bar{H}_{f 2}^{+}(\omega)=-\sin \frac{\omega}{V} a_{b} \sin \frac{\omega}{V} a_{c} ;(1)$ 
- for the antisymmetrical excitation modes

$$
\bar{H}_{f 1}^{-}=i \cos \frac{\omega}{V} a_{b} \sin \frac{\omega}{V} a_{c} ; \bar{H}_{f 2}^{-}=i \sin \frac{\omega}{V} a_{b} \cos \frac{\omega}{V} a_{c}
$$

The functions above help determine the filtering speeds corresponding to the geometric filtering effect, which comes from the distance between the bogie axles, as well as from the geometric filtering effect due to the distance between the bogies. Thus, for the excitation frequency $f=\omega / 2 \pi$, and for the speeds calculated via the relation

$V=\frac{4 a_{b} f}{2 n+1}$, with $n=0,1$

the symmetrical and antisymmetrical bounce in the axles' planes is completely filtered by the distance between the bogie axles and the geometric filtering effect due to the bogie wheelbase.

As for the symmetrical and antisymmetrical pitch in the axles' planes, these excitation modes are filtered by the distance between the bogie axles at the speeds of

$$
V=\frac{2 a_{b} f}{n}, \text { where } n=1,2
$$

The distance between the bogies also filters the excitations due to the bounce and pitch motions in the axles' planes and the geometric filtering effect from the carbody wheelbase, where this effect has a maximum efficiency at the speeds

$$
V=\frac{4 a_{c} f}{2 n+1}, \text { with } n=0,1,2, \ldots,
$$

for the symmetrical bounce mode and antisymmetrical pitch in the axles' planes and at the speeds of

$$
V=\frac{2 a_{c} f}{n}, \text { where } n=1,2, \ldots
$$

for the antisymmetrical bounce mode and symmetrical pitch.

When taking into account a certain excitation frequency, the maximum geometric filtering speeds can be calculated, by means of the equations mentioned above.

Table 3. The maximum geometric filtering velocities corresponding to the effect given by the bogie wheelbase

\begin{tabular}{|l|l|l|l|}
\hline \multirow{2}{*}{$\begin{array}{l}\text { Excitation frequency } \\
\text { Excitation modes }\end{array}$} & $\begin{array}{l}1.17 \\
\mathrm{~Hz}\end{array}$ & $\begin{array}{l}8.20 \\
\mathrm{~Hz}\end{array}$ & $\begin{array}{l}1.46 \\
\mathrm{~Hz}\end{array}$ \\
\cline { 2 - 4 } & \multicolumn{2}{|l|}{$\begin{array}{l}\text { Maximum filtering velocity } \\
{[\mathrm{km} / \mathrm{h}]}\end{array}$} \\
\hline $\begin{array}{l}\text { Symmetrical and } \\
\text { antisymmetrical bounce in } \\
\text { the axles' plans }\end{array}$ & 21.56 & 151.14 & 26.91 \\
\hline $\begin{array}{l}\text { Symmetrical and } \\
\text { antisymmetrical pitch in } \\
\text { the axles' plans }\end{array}$ & 10.78 & 75.57 & 13.45 \\
\hline
\end{tabular}

Table 4. The maximum geometric filtering velocities corresponding to the effect given by the carbody wheelbase

\begin{tabular}{|l|l|l|l|}
\hline $\begin{array}{l}\text { Exeitation frequency } \\
\text { Excitation modes }\end{array}$ & $\mathrm{Hz}$ & \multicolumn{2}{|l|}{$\mathrm{Hz}$} \\
\cline { 2 - 5 } & $\begin{array}{l}\text { Maximum filtering velocity } \\
{[\mathrm{km} / \mathrm{h}]}\end{array}$ & \\
\hline $\begin{array}{l}\text { Symmetrical bounce and } \\
\text { antisymmetrical pitch in } \\
\text { the axles' plans }\end{array}$ & 160 & 1121.16 & 199.72 \\
\hline $\begin{array}{l}\text { Antisymmetrical bounce } \\
\text { and symmetrical pitch in } \\
\text { the axles' plans }\end{array}$ & 80 & 560.08 & 99.86 \\
\hline
\end{tabular}

The tables 3 and 4 show the maximum geometric filtering speeds corresponding to the effect given by the bogie wheelbase and the carbody wheelbase respectively, for the resonance frequencies of the carbody bounce, symmetrical bending and pitch. What can be firstly noticed is that the carbody wheelbase triggers a geometric filtering effect on a larger speed interval than the one corresponding to the filtering effect from the bogie wheelbase. Secondly, the geometric filtering effect is also visible up to high and very high velocities at the frequency of $8.2 \mathrm{~Hz}$ only, provided that this effect comes from the carbody wheelbase. Another observation would be that the bogie wheelbase produces filtering effect at only low velocities, for the frequencies of $1.17 \mathrm{~Hz}$ and $1.46 \mathrm{~Hz}$.
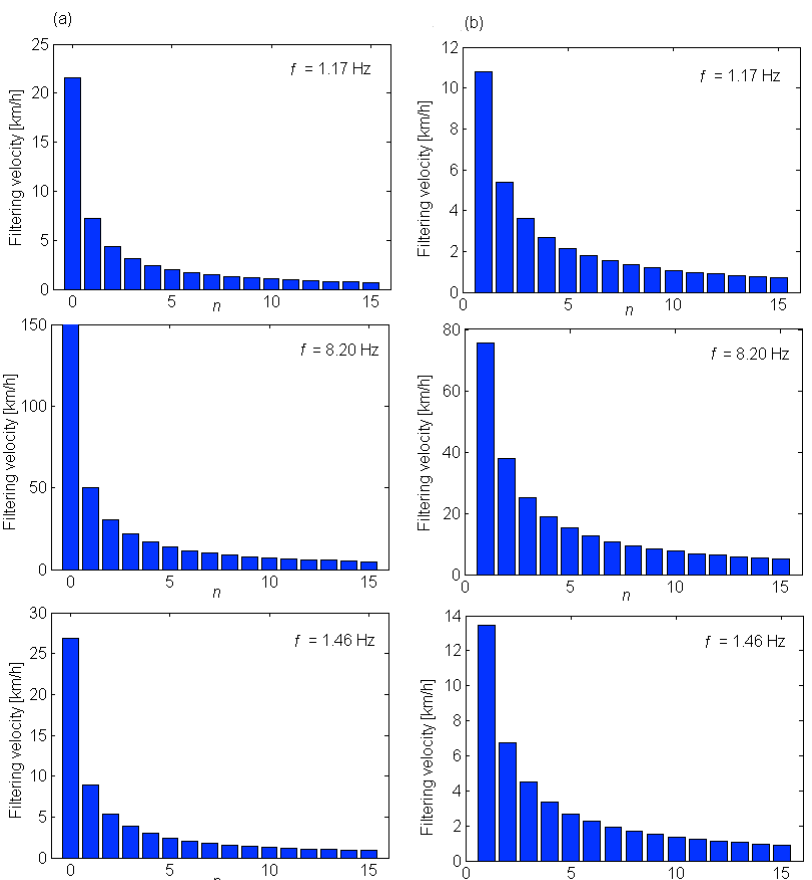

Fig. 4. Filtering velocities corresponding to the geometric filtering effect given by the bogie wheelbase (a) for the symmetrical and antisymmetrical bounce in the axles' planes; (b) for the symmetrical and antisymmetrical pitch in the axles' planes.

The figures 4 and 5 feature the filtering velocities for the resonance frequencies of the carbody bounce, symmetrical bending and pitch, calculated by means of relations (3) - (6), where $n_{\max }=15$. What can be noticed in the diagrams in figure 4, as shown above, is that the bogie wheelbase triggers a filtering effect at only very low velocities at frequencies of $1.17 \mathrm{~Hz}$ and $1.46 \mathrm{~Hz}$. Moreover, the filtering velocities at frequency of $8.20 \mathrm{~Hz}$ are being analyzed. Except for the maximum filtering velocity, the filtering velocities of the symmetrical and antisymmetrical bounce in the axles' planes are lower than $50 \mathrm{~km} / \mathrm{h}$. Likewise, when the maximum filtering velocity is disregarded, the filtering 
velocities of the symmetrical and antisymmetrical pitch in the axles' planes do not exceed $40 \mathrm{~km} / \mathrm{h}$.
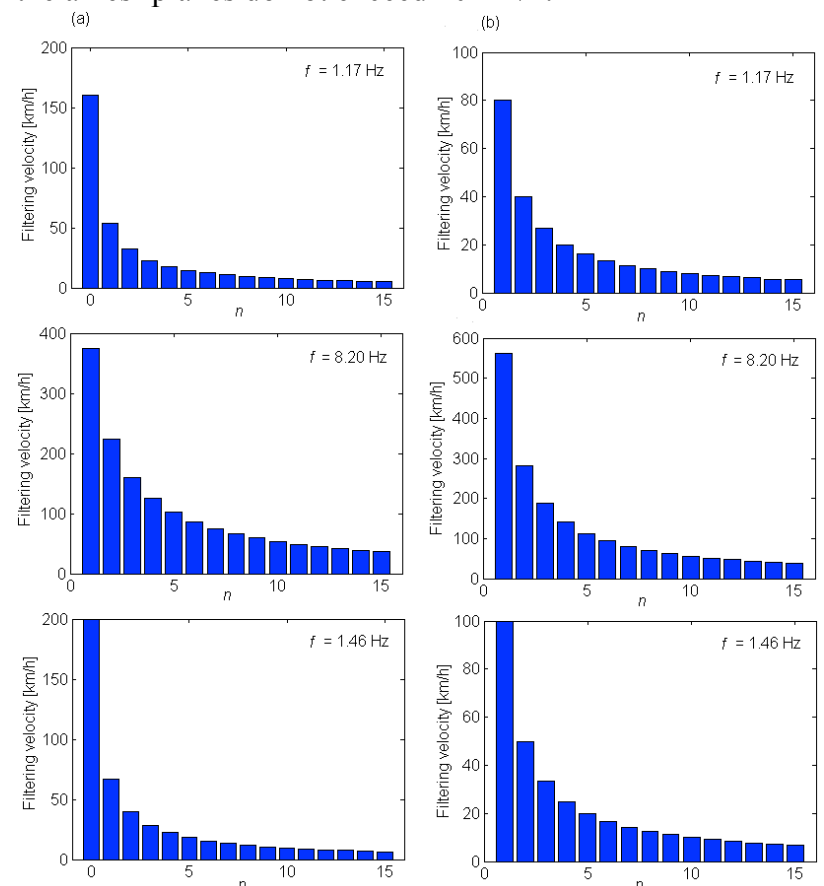

Fig. 5. Filtering velocities corresponding to the filtering effect given by the carbody wheelbase: (a) for the symmetrical bounce and antisymmetrical pitch in the axles' planes; (b) for the antisymmetrical bounce and the symmetrical pitch in the axle's planes.

Based on the diagrams in figure 5, the domain of the filtering velocities corresponding to the filtering effect given by the carbody wheelbase will be examined, while excluding the maximum filtering velocities. Thus, at the filtering velocities of the symmetrical bounce and antisymmetrical pitch in the axles' planes, at frequencies of $1.17 \mathrm{~Hz}$ and 1.46 $\mathrm{Hz}$, can be demonstrated that they do not exceed the values of $55 \mathrm{~km} / \mathrm{h}$ and $65 \mathrm{~km} / \mathrm{h}$ respectively, as most of them are even less than $20 \mathrm{~km} / \mathrm{h}$. Similar observations can be made about the filtering velocities of the antisymmetrical bounce and symmetrical pitch in the axles' planes, namely that the filtering velocities are smaller than $40 \mathrm{~km} / \mathrm{h}$ and $50 \mathrm{~km} / \mathrm{h}$; most filtering velocities are included in the range of very small velocities. In terms of the filtering velocities at the frequency of $8.20 \mathrm{~Hz}$, they are noticed to cover a large interval, which goes up to $375 \mathrm{~km} / \mathrm{h}$ for the symmetrical bounce and the antisymmetrical pitch in the axles' planes and to $280 \mathrm{~km} / \mathrm{h}$ for the antisymmetrical bounce and the symmetrical pitch in the same planes.

Further on, the response functions of the vehicle are analyzed in the carbody reference points, at the resonance frequencies of its main motions - bounce, symmetrical bending and the pitch.
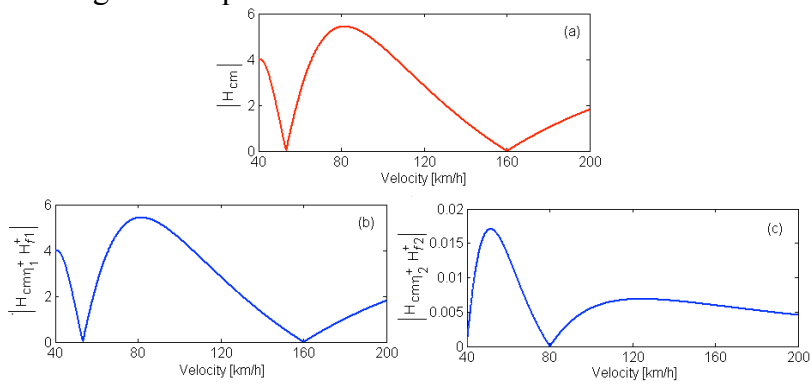

Fig. 6. The response functions at carbody centre at frequency of 1.17 $\mathrm{Hz}$.
Figure 6 includes the response functions calculated at the carbody centre, frequency of $1.17 \mathrm{~Hz}$ (diagram (a)) and the partial response functions weighted by the corresponding filtering characteristics, namely: the partial response function to the excitation due to the symmetrical bounce in the axles' planes (diagram (b)) and the partial response function to the excitation coming from the symmetrical pitch in the axles' planes (diagram (c)). The examination of the three diagrams leads to the conclusion that the vehicle response is prompted by the partial response to the excitation due to the symmetrical bounce in the axles' planes. The diagram (a) identifies two minimum values corresponding to the geometric filtering effect of the symmetrical bounce in the axles' planes (fig 3, diagram (a)). They are recorded against the filtering velocities correlative with the effect given by the carbody wheelbase, at $53.4 \mathrm{~km} / \mathrm{h}$ and $160 \mathrm{~km} / \mathrm{h}$.
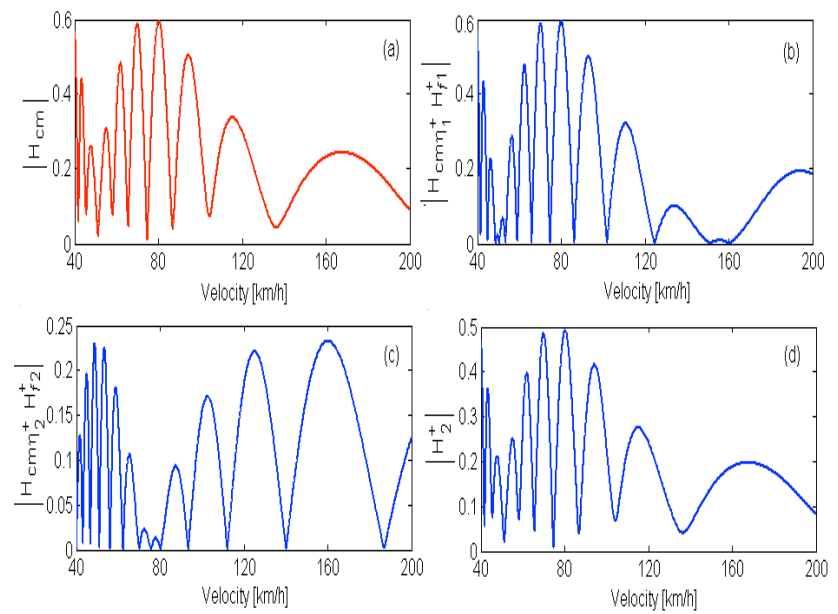

Fig. 7. The response functions at carbody centre for frequency of 8.2 $\mathrm{Hz}$.

The analysis of the response at the carbody centre at frequency of $8,20 \mathrm{~Hz}$ relies on the diagrams in fig. 7, which features the response function $\bar{H}_{c m}$ (diagram (a)) and the partial response functions to the excitation from symmetrical bounce in the axles' planes $\bar{H}_{c m \eta_{1}^{+}}$(diagram (b)), and the excitation due to the symmetrical pitch in the same plans $\bar{H}_{c m \eta_{2}^{+}}$(diagram (c)), weighted by the corresponding

filtering characteristics. The observation is that the response at the carbody centre is mainly triggered by the symmetrical pitch in the axles' planes and to a smaller extent by the excitation coming from the symmetrical bounce in the same planes. For that matter, diagram (d) shows that the response at the carbody centre is practically the result of the symmetrical bending excited by both the symmetrical bounce and also by the symmetrical pitch in the axles' planes. The carbody response shows more minimum values corresponding to the geometric filtering effect of the symmetrical bounce combined with the same effect of the symmetrical pitch in the axles' plans. Due to this fact, the minimum values of the response function occur at velocities that are different from the filtering ones for the two excitation modes $(41.8 \mathrm{~km} / \mathrm{h} ; 45.6 \mathrm{~km} / \mathrm{h} ; 51.2 \mathrm{~km} / \mathrm{h} ; 58.1$ $\mathrm{km} / \mathrm{h} ; 65.5 \mathrm{~km} / \mathrm{h} ; 74.7 \mathrm{~km} / \mathrm{h} ; 86.9 \mathrm{~km} / \mathrm{h} ; 104.4 \mathrm{~km} / \mathrm{h} ; 136$ $\mathrm{km} / \mathrm{h})$. 

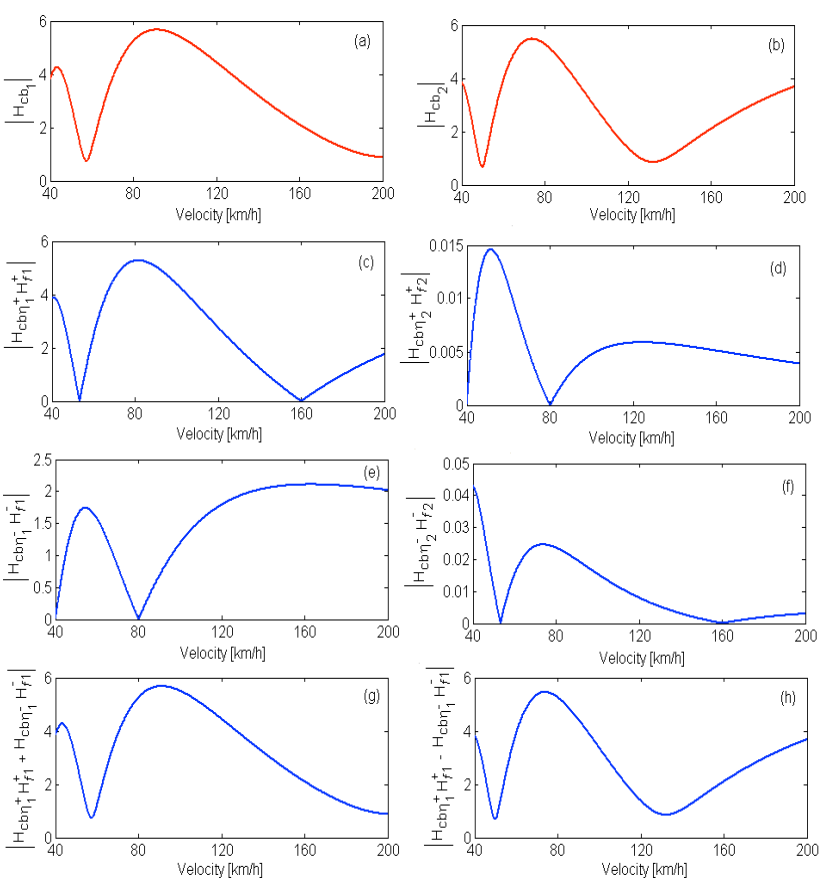

Fig. 8. The response functions above the bogies at frequency of 1.17 $\mathrm{Hz}$.

The figure 8 features the response functions in the carbody reference points located above the two bogies (diagrams (a) and (b)) at the bounce resonance frequency $(1.17 \mathrm{~Hz})$. The diagrams (c) and (d) are the partial response functions to the symmetrical excitation modes and the diagrams (e) and (f) are the partial response functions to the antisymmetrical excitation modes, weighted by the corresponding filtering characteristics. Thus, it is evident that the carbody response in the points above the bogies is mainly the result of the overlapping of the partial response function to the excitation from the symmetrical bounce in the axles' planes and of the partial response function to the excitation due to the antisymmetrical bounce in the axles' planes (diagrams (g) and (h)). Under these conditions, it is about a filtering characteristic given by the combined filtering effect of the symmetrical bounce with the antisymmetrical bounce in the axles' planes. This filtering characteristic introduces minimum values in the carbody response at velocities different from the filtering velocities of the two excitation modes. As for the response function of the carbody above the front bogie, there is only a minimum value at speed $57.4 \mathrm{~km} / \mathrm{h}$, while the rear bogie will have two minimum values, at $50 \mathrm{~km} / \mathrm{h}$ and at $132 \mathrm{~km} / \mathrm{h}$.

Similar conclusions with the above will be drawn for the resonance frequency of the carbody pitch $(1.46 \mathrm{~Hz})$, based on the diagrams in fig. 9. At this frequency, the response in the carbody reference points above the bogies (diagrams (a) and (b)) is also noticed to be the result of the overlapping of the partial response to the excitation due to the symmetrical bounce in the axles' planes and of the partial response from the excitation derived from the antisymmetrical bounce in the same planes (diagrams (g) and (h)). The filtering characteristic given by the combined filtering effect of the two excitation modes triggers minimum values of the response function above the front bogie at velocities of 47.1 $\mathrm{km} / \mathrm{h}$ and $88.8 \mathrm{~km} / \mathrm{h}$, and at velocities of $53.5 \mathrm{~km} / \mathrm{h}$ and 115 $\mathrm{km} / \mathrm{h}$ above the rear bogie.
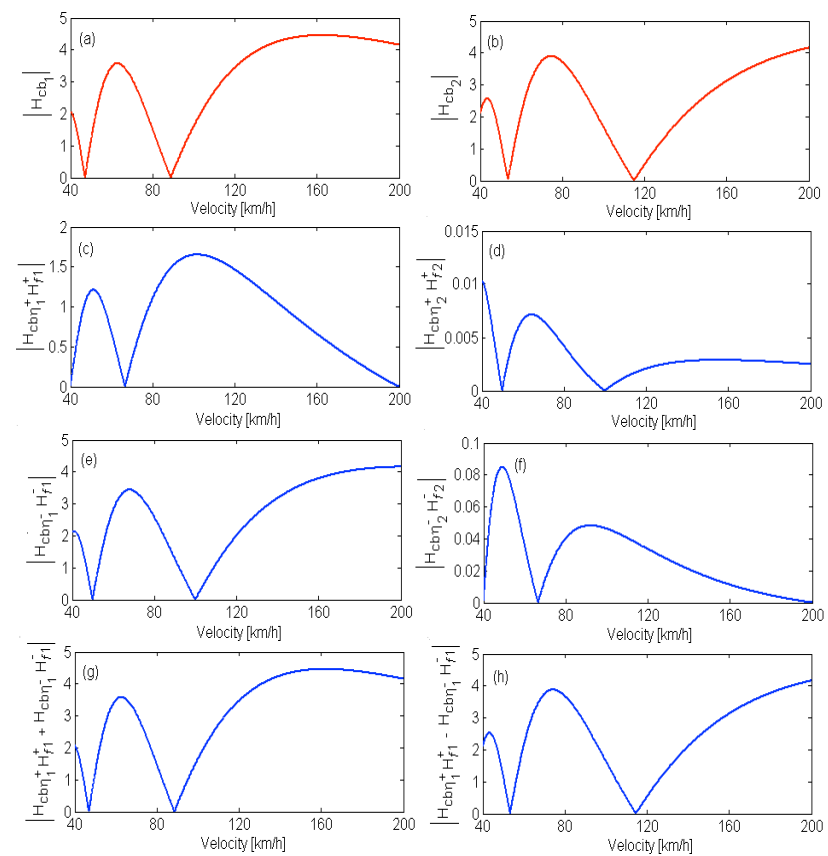

Fig. 9. The response functions above the bogies at frequency of 1.46 $\mathrm{Hz}$.
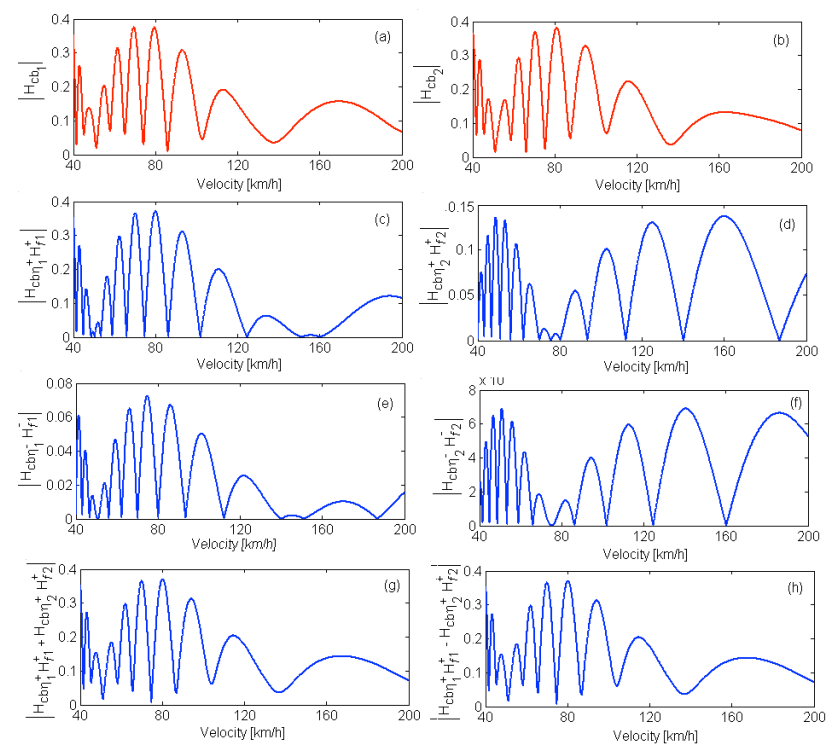

Fig. 10. The response functions above the bogies at frequency of 8.2 $\mathrm{Hz}$.

Figure 10 features the response functions in the carbody reference points above the bogies, for the frequency of 8.20 $\mathrm{Hz}$. In this case, the carbody response is mainly due to the contribution of two partial response functions, according to the diagrams (g) and (h). It is about the partial response functions to the symmetrical excitation modes. Each of these partial response functions are weighted by a filtering characteristic - the filtering characteristic of the symmetrical bounce in the axles' planes and the filtering characteristic of the symmetrical pitch in the same planes, respectively, which shows certain filtering velocities (see fig. 3, diagrams (b)). The filtering characteristic of the carbody response function above the bogies does not maintain the properties of the two filtering characteristics and this fact is obvious from the fact that the velocities where minimum values of the functions $\bar{H}_{c b_{1,2}}$ occur no longer correspond to the filtering velocities of the symmetrical bounce and pitch in the axles' 
planes. For instance, the carbody response function against the front bogie records minimum values at the speeds of 41.5 $\mathrm{km} / \mathrm{h} ; 45.2 \mathrm{~km} / \mathrm{h} ; 51.3 \mathrm{~km} / \mathrm{h} ; 57.9 \mathrm{~km} / \mathrm{h} ; 65.2 \mathrm{~km} / \mathrm{h} ; 74.2$ $\mathrm{km} / \mathrm{h} ; 86 \mathrm{~km} / \mathrm{h} ; 102.8 \mathrm{~km} / \mathrm{h} ; 137.5 \mathrm{~km} / \mathrm{h}$.

\section{The analysis of the vehicle dynamic response to the track stochastic irregularities}

This section deals with the analysis of the dynamic response of the vehicle to the track stochastic irregularities and of the influence of the geometric filtering effect upon the ride quality, evaluated as based on the root mean square of the vertical acceleration [4] and on the ride comfort estimated by means of the comfort index [11].
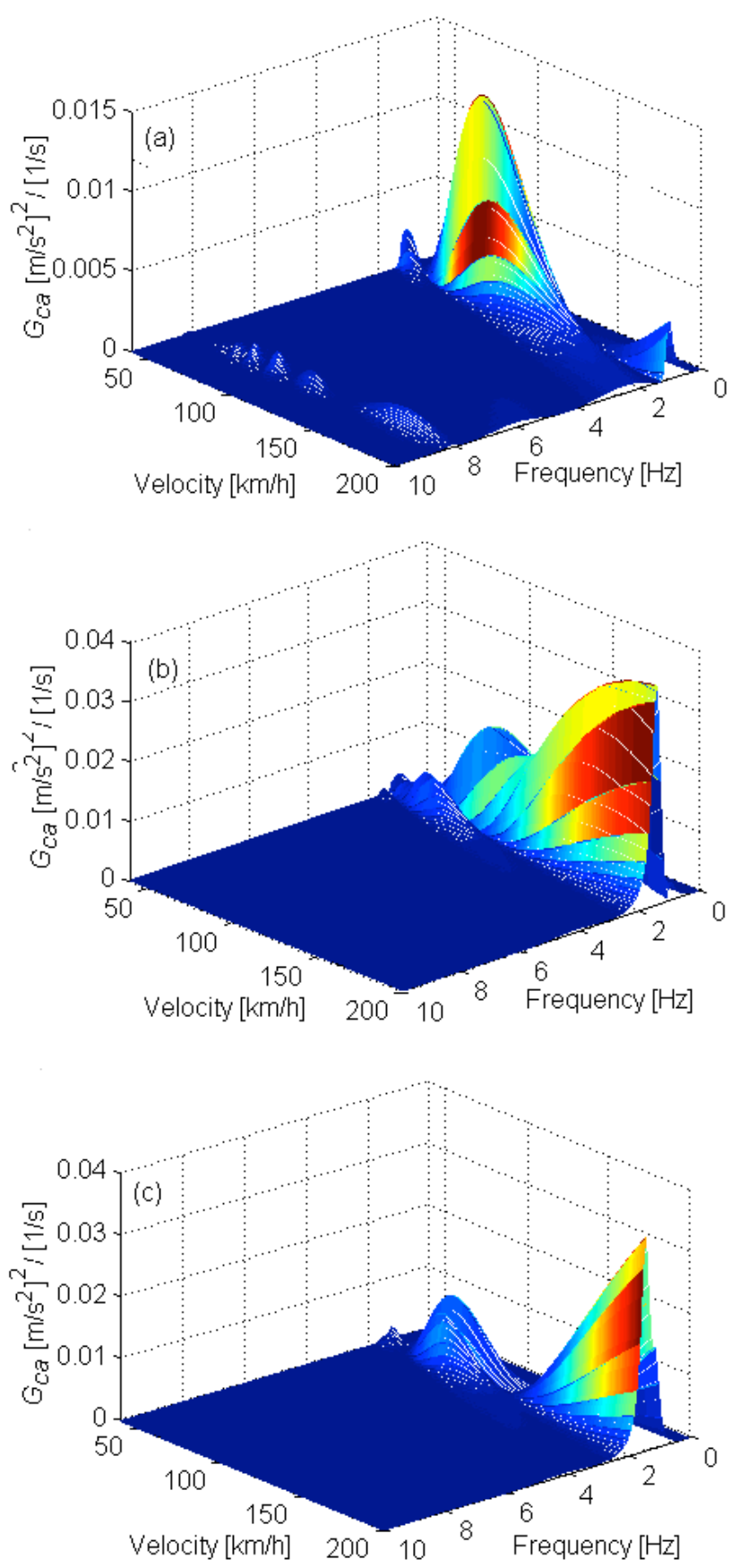

Fig. 11. Acceleration power spectral density: (a) at the carbody centre; (b) above the front bogie; (c) above the rear bogie
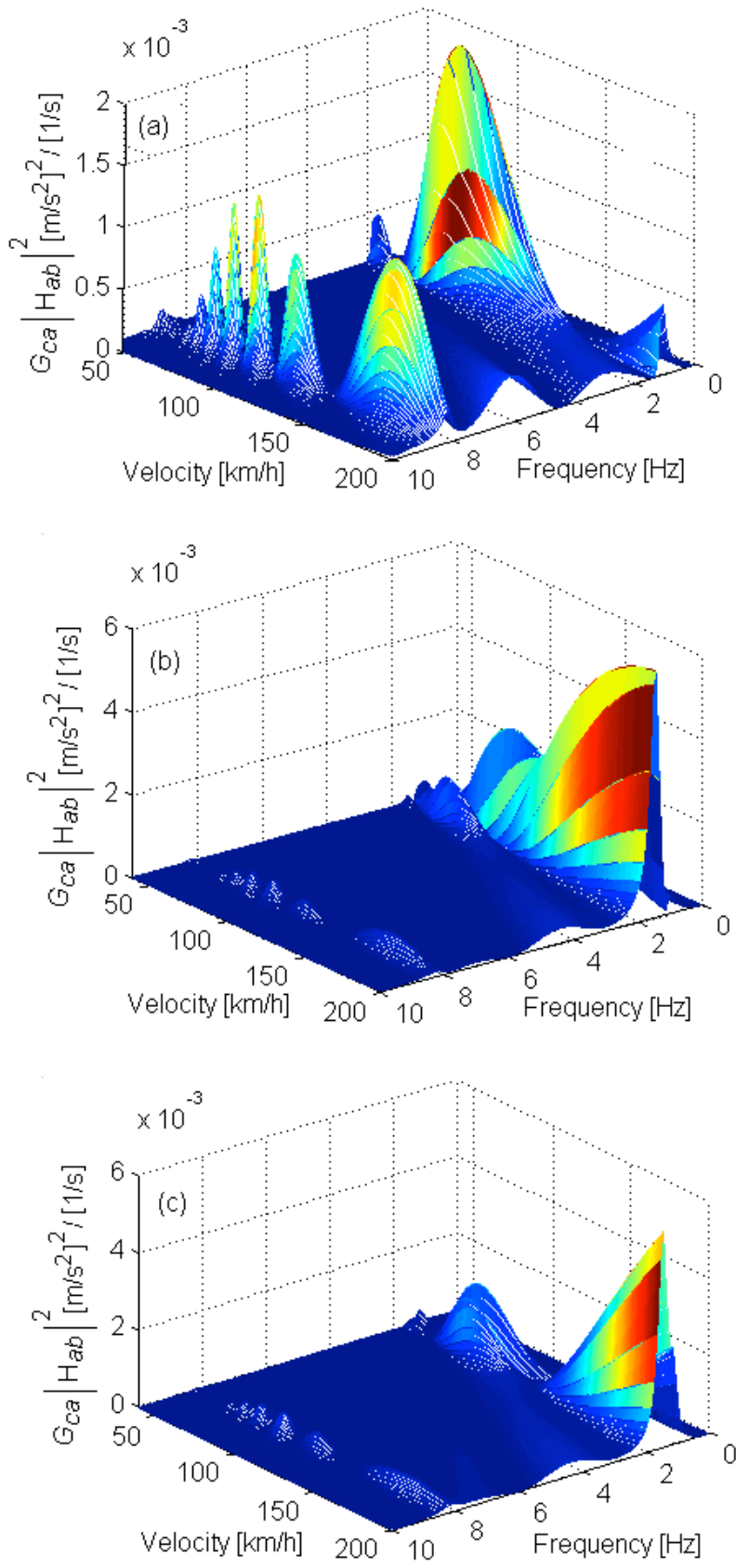

Fig. 12. Power spectral density of the balanced acceleration: (a) at the carbody centre; (b) above the front bogie; (c) above the rear bogie

The dynamic response of the vehicles is expressed by the power spectral density of the carbody vertical acceleration, calculated in its reference points (see fig. 11). There will be considered the frequency range that includes the resonance frequencies of the main carbody motions $(0 \ldots 10 \mathrm{~Hz})$ and the velocity interval of $40 \ldots 200 \mathrm{~km} / \mathrm{h}$. At the carbody centre, the power spectral density of the acceleration is dominated by the carbody bounce at $1.17 \mathrm{~Hz}$ on a wide range of velocities (diagram (a)). There are also obvious the peaks corresponding to the carbody symmetrical bending at $8.20 \mathrm{~Hz}$, where this vibration mode has a higher importance around the velocity of ${ }^{\prime} 170 \mathrm{~km} / \mathrm{h}$. Apart from the peaks correlative with the resonance frequencies of the two vibration modes, a series of minimum values are evident, corresponding to the geometric filtering effect [12]. For instance, at frequency $1.17 \mathrm{~Hz}$, these minimum values can be found at the filtering velocities of $53.4 \mathrm{~km} / \mathrm{h}$ and 160 $\mathrm{km} / \mathrm{h}$. 
Above the bogies, the dominant vibration modes are the bounce at $1.17 \mathrm{~Hz}$ and the pitch, at $1.46 \mathrm{~Hz}$. For these frequencies, the power spectral density of the acceleration goes up along the velocity, but this increase is not continuous, due to the geometric filtering effect, which introduces a series of minimum values. As an example, the power spectral density of the acceleration is minimum at speed $57.4 \mathrm{~km} / \mathrm{h}$ for the frequency of $1.17 \mathrm{~Hz}$ above the front bogie, whereas this value is recorded at speeds of 47.1 $\mathrm{km} / \mathrm{h}$ and $88.8 \mathrm{~km} / \mathrm{h}$ for the frequency of $1.46 \mathrm{~Hz}$. In the carbody reference point located above the rear bogie, the power spectral density of the acceleration is minimum in the following situations: for frequency of $1.17 \mathrm{~Hz}$, at velocities of $50 \mathrm{~km} / \mathrm{h}$ and $132 \mathrm{~km} / \mathrm{h}$; for frequency of $1.46 \mathrm{~Hz}$, at velocities of $53.5 \mathrm{~km} / \mathrm{h}$ and $115 \mathrm{~km} / \mathrm{h}$. What needs to be noticed is that these speed values to which the vehicle response to the track stochastic irregularities is minimum have been also identified during the analysis of the carbody response functions (see fig. 8 - diagrams (a) and (b) and fig. 9 - diagrams (a) and (b)).

The figure 12 shows the power spectral density of the carbody vertical acceleration, weighted by the transfer function of the acceleration weighting filter $\left(W_{a b}=W_{a} \cdot W_{b}\right)$, in order to calculate the comfort index. It should be reminded that the filter $W_{a}$ is a band-pass type filter, while $W_{b}$ is a weighting filter that takes into account the high sensitivity of the human body to the vertical vibrations within the frequency interval of $3 \ldots 13 \mathrm{~Hz}$ [11]. In the light of the above, an explanation can be given for the amplification of the carbody dynamic response to the frequency of $8.20 \mathrm{~Hz}$, mainly at its centre where this vibration mode has a higher influence than above the bogies. Likewise, for velocities beyond $180 \mathrm{~km} / \mathrm{h}$, the increase of the acceleration power spectral density should be noticed against the resonance frequency of the bogie bounce motion $(6.6$ $\mathrm{Hz}$ ). The diagram of the power spectral density of the weighted acceleration also emphasizes clearly the geometric filtering effect, which is visible at frequency of $8.20 \mathrm{~Hz}$.
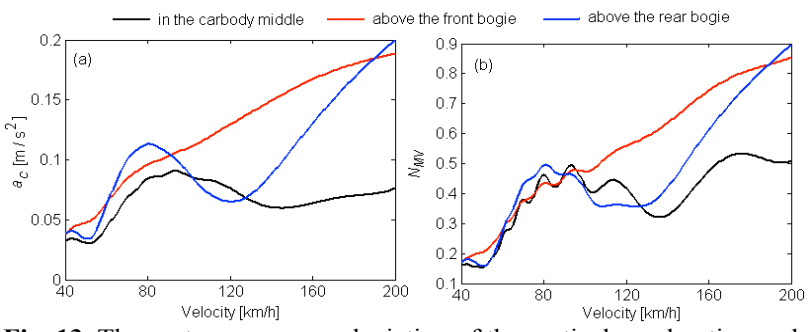

Fig. 13. The root mean square deviation of the vertical acceleration and the comfort index.

Figure 13 features the root mean square of the vertical acceleration (diagram (a)) and the comfort index (diagram (b)), calculated in the carbody reference points, for velocities between 40 and $200 \mathrm{~km} / \mathrm{h}$. A former observation would be in regards to the fact that the root mean square of the acceleration is generally smaller at the carbody centre and goes up above the two bogies. For the comfort index, this observation applies for velocities higher than $130 \mathrm{~km} / \mathrm{h}$. Up to this speed, the comfort index at the carbody centre has close values or even higher than the comfort index calculated above the two bogies. A latter observation is that both acceleration and the comfort index mostly increase along with the velocity, but not in a continuous manner. For certain velocities, sudden decreases of the two occur, due to the geometric filtering effect.
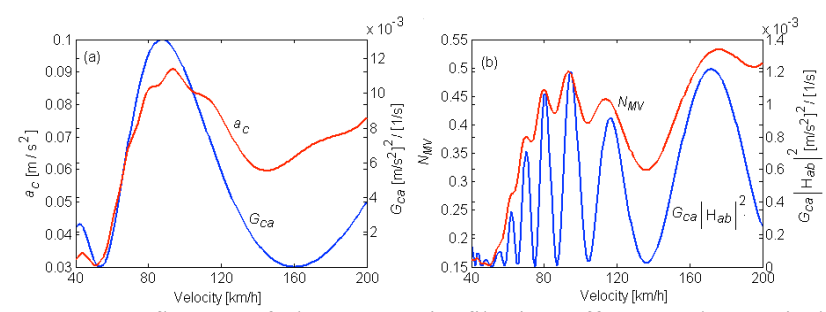

Fig. 14. Influence of the geometric filtering effect on the vertical acceleration and on the comfort index at the carbody centre

Upon examining the root mean square acceleration at the carbody centre can be indeed noticed that it maintains the characteristics of the power spectral density of the acceleration at frequency of $1.17 \mathrm{~Hz}$ (fig. 14, diagram (a)). As a consequence, the decline of the root mean square acceleration at the velocities of $51 \mathrm{~km} / \mathrm{h}$ and $145 \mathrm{~km} / \mathrm{h}$ happens because of the geometric filtering effect of the symmetrical bounce in the axles' planes. Moreover, for the speed interval of $80 \mathrm{~km} / \mathrm{h} \ldots .120 \mathrm{~km} / \mathrm{h}$, it becomes evident the influence of the combined geometric filtering effect corresponding to the symmetrical bounce and the symmetrical pitch in the axles' planes, which is visible at the carbody centre at the frequency of $8.20 \mathrm{~Hz}$.

In terms of the comfort index, the distribution of this unit as a speed function shows the characteristics of the power spectral density of the weighted acceleration with the transfer function of the filter $W_{a b}$, calculated at frequency of $8.20 \mathrm{~Hz}$ (fig. 14, diagram (b)). Therefore, it can be inferred that the decrease of the comfort index for certain velocities is due to the combined geometric filtering effect corresponding to the symmetrical bounce and the symmetrical pitch in the axles' planes that are visible at the carbody centre for the frequency of $8.20 \mathrm{~Hz}$.

\section{Conclusions}

The objective of the Part II in this paper has been the numerical analysis of the dynamic response of the railway vehicle to the track vertical irregularities. In this context, while considering the three carbody reference points - at the centre and above the bogies - the response functions to the symmetrical and antisymmetrical excitation modes have been examined, as induced by the track vertical irregularities, as well as the geometric filtering effect characteristics of these excitation modes. The results herein featured have shown a series of properties relevant for the vertical vibrations behaviour of the vehicle. Thus, based on the analysis of the partial response functions, the vibration modes prevailing in the vehicle response have been presented to be dependent on the reference point and on the excitation mode. Likewise, the vibration modes that are significant in the carbody response are as such: the carbody bounce - in the reference point located at the carbody centre; the carbody bounce and pitch - in the reference points above the bogies.

Upon examining the geometric filtering effect characteristics of the symmetrical and antisymmetrical excitation modes, as well as of the geometric filtering velocities, at the resonance frequencies of the main carbody motions, the following conclusions have been reached to:

- the geometric filtering effect has a selective nature, depending on the velocity and the excitation frequency; this is more visible at smaller velocities and higher excitation frequencies; 
- the geometric filtering effect coming from the carbody wheelbase manifests itself on a larger velocity interval than the one corresponding to the filtering effect from the bogie wheelbase.

In correlation with the previous observations, the geometric filtering effect can be noticed to being more efficient at the resonance frequency of the carbody symmetrical bending $(8.20 \mathrm{~Hz})$. For this frequency, the filtering effect shows up to high and very high velocities, should this effect comes from the carbody wheelbase.

The analysis of the response functions in the carbody reference points at the resonance frequencies of its main motions has led to the conclusions regarding the contributions of the partial response functions corresponding to each excitation mode (symmetrical or antisymmetrical bounce in the axles' planes; symmetrical or antisymmetrical pitch in the same planes). It has been demonstrated that the carbody response can be determined by a single partial response function, as with the response at the carbody centre at frequency of $1.17 \mathrm{~Hz}$, which is given by the partial response function to the excitation due to the symmetrical bounce in the axles' planes. In this case, the carbody response is only affected by the filtering effect of the symmetrical bounce in the axles' planes; the response function is minimum at the filtering velocities corresponding to this filtering effect. The carbody response can also be the result of the contribution of two partial response functions, as it happens with the response at the carbody centre at frequency of $8.20 \mathrm{~Hz}$ and above the bogies, for all three frequencies being considered. For instance, the response above the bogies at frequencies of $1.17 \mathrm{~Hz}$ and $1.46 \mathrm{~Hz}$ is the result of the overlapping of the partial response to the excitation coming from the symmetrical bounce in the axles' planes and of the partial response to the excitation from the antisymmetrical bounce in the same planes; at the frequency of $8.20 \mathrm{~Hz}$, the response comes from the partial response functions to the symmetrical excitation modes. Under these circumstances, the filtering characteristic of the carbody response function is the result of the combined filtering effect of two excitation modes. Consequently, the velocities that record minimum values of the carbody response functions are different from the filtering velocities corresponding to the two excitation modes.

The analysis of the vehicle dynamic response to the track stochastic irregularities, expressed by the power spectral density of the vertical acceleration calculated in the carbody reference points has pointed out at a series of aspects that have been emphasized on during the analysis of the carbody response functions. It is about the vibration modes that are dominant in the spectrum of the acceleration power spectral density, namely: bounce - at the carbody centre; bounce and pitch - above the bogies. Similarly, the focus has been placed on the influence of the geometric filtering effect that triggers minimum values of the power spectral density of acceleration at certain velocities. Moreover, the carbody dynamic response has been shown to amplify at frequency of $8.20 \mathrm{~Hz}$, mainly at its centre where this vibration mode has a higher influence than against the bogie, by means of the weighting of the acceleration power spectral density via a filter that considers the increased sensitivity to the vertical vibrations within the frequency range of $3 \ldots . .13 \mathrm{~Hz}$.

To have the analysis of how the geometric filtering effect influences the ride quality and the ride comfort of the vehicle, the root mean square of the acceleration and the comfort index have been calculated in the carbody reference points. The distribution of these units as speed functions has been demonstrated to have minimum local values, due to the geometric filtering effect of the excitation modes that trigger the carbody dynamic response in its reference points.

\section{References}

1. J. Evans, M. Berg, Challenges in simulation of rail vehicle dynamics, Vehicle System Dynamics, 47, 1023-1048, 2009.

2. G. Schupp, Simulation of railway vehicles: Necessities and applications, Mechanics Based Design of Structures and Machines, 31, 3, 297-314, 2003.

3. S. Iwnicki, Handbook of railway vehicle dynamics, CRC Press Taylor \& Francis Group, 2006.

4. UIC 518 Leaflet - 4th edition - Sept. 2009. Testing and approval of railway vehicles from the point of view of their dynamic behaviour - Safety - Track fatigue - Running behaviour.

5. EN 14363 - 2013. Railway applications - Testing and Simulation for the acceptance of running characteristics of railway vehicles Running behaviour and stationary tests.

6. C. Funfschilling, Y. Bezin, M. Sebès, DynoTRAIN: Introduction of simulation in the certification process of railway vehicles, Transport Research Arena, Paris, Paper 19829, 2014.
7. C. Funfschilling, G. Perrin, S. Kraft, Propagation of variability in railway dynamic simulations: Application to virtual homologation, Vehicle System Dynamics, 50, Supplement, 245-261, 2012.

8. I. Sebeşan, T. Mazilu, Vibraţiile vehiculelor feroviare (Vibrations of the railway vehicles), MatrixRom, București, 2010.

9. M. Dumitriu, Geometric filtering effect of vertical vibrations of railway vehicles, Analele Universităţii "Eftimie Murgu" Resița, 1, 48-61, 2012.

10. M. Dumitriu, Considerations on the geometric filtering effect of the bounce and pitch movements in railway vehicles, Annals of Faculty Engineering Hunedoara - International Journal of Engineering, XII, 3, 155-164, 2014.

11. ENV 12299/1997, Railway applications ride comfort for passengers measurement and evaluation.

12. M. Dumitriu, Influence of the vertical suspension on the vibration behavior in the railway vehicles, Annals of the University of Petroşani, Mechanical Engineering, 13, 35-50, 2011. 